\title{
Recurrence relations and outer relative asymptotics of orthogonal polynomials with respect to a discrete Sobolev type inner product
}

\author{
Francisco Marcellán • M. Francisca Pérez-Valero • \\ Yamilet Quintana • Alejandro Urieles
}

Received: 8 August 2013 / Revised: 14 October 2013 / Accepted: 16 October 2013 /

Published online: 26 October 2013

(C) The Author(s) 2013. This article is published with open access at SpringerLink.com

Abstract We investigate algebraic and analytic properties of sequences of polynomials orthogonal with respect to the Sobolev type inner product

$$
\langle f, g\rangle=\int f(x) g(x) d \mu(x)+\sum_{k=1}^{K} \sum_{i=0}^{N_{k}} M_{k, i} f^{(i)}\left(b_{k}\right) g^{(i)}\left(b_{k}\right)
$$

where $\mu$ is a finite positive Borel measure belonging to the Nevai class, the mass points $b_{k}$ are located outside the support of $\mu$, and $M_{k, i}$ are complex numbers such that $M_{k, N_{k}} \neq 0$. First, we study the existence as well as recurrence relations for such

Communicated by S. K. Jain.

The second author was partially supported by the Research Fellowship Program from Ministerio de Ciencia e Innovación (MTM 2009-12740-C03-01), Spain. The first, second and third authors were partially supported by a grant from Ministerio de Economía y Competitividad. Dirección General de Investigación Científica y Técnica (MTM 2012-36732-C03-01), Spain.

F. Marcellán · M. F. Pérez-Valero

Departamento de Matemáticas, Universidad Carlos III de Madrid,

Avenida de la Universidad 30, 28911 Leganés, Madrid, Spain

e-mail: pacomarc@ing.uc3m.es

M. F. Pérez-Valero

e-mail: mpvalero@math.uc3m.es

Y. Quintana $(\varangle) \cdot$ A. Urieles

Departamento de Matemáticas Puras y Aplicadas, Edificio Matemáticas y Sistemas (MYS),

Universidad Simón Bolívar, Apartado Postal: 89000, Caracas 1080 A, Venezuela

e-mail: yquintana@usb.ve

A. Urieles

e-mail: aurielesg@gmail.com 
polynomials. When the values $M_{k, i}$ are nonnegative real numbers, we can deduce the coefficients of the recurrence relation in terms of the connection coefficients for the sequences of polynomials orthogonal with respect to the Sobolev type inner product and those orthogonal with respect to the measure $\mu$. The matrix of a symmetric multiplication operator in terms of the above sequence of Sobolev type orthogonal polynomials is obtained from the Jacobi matrix associated with the measure $\mu$. Finally, we focus our attention on some outer relative asymptotics of such polynomials, which are deduced by using the above connection formulas.

Keywords Nevai class - Sobolev inner products - Orthogonal polynomials · Recurrence relations · Connection coefficients · Outer relative asymptotics.

\section{Mathematics Subject Classification (2000) $\quad 42 \mathrm{C} 10 \cdot 33 \mathrm{C} 45$}

\section{Introduction}

The study of asymptotic properties for general orthogonal polynomials is an important challenge in approximation theory and their applications permeate many fields in science and engineering $[16,25,26]$. Although it may seem an old subject from the point of view of the standard orthogonality [26], this is not the case neither in the general setting (cf. $[9,10,16,21-23,25])$ nor from the point of view of Sobolev orthogonality, where it remains like a partially explored subject [3]. In fact, new results continue to appear in some recent publications [4,6-8, 11-15, 18-20].

A discrete Sobolev-type inner product is defined by

$$
\langle f, g\rangle=\int f(x) g(x) d \mu(x)+\sum_{k=1}^{K} \sum_{i=0}^{N_{k}} M_{k, i} f^{(i)}\left(b_{k}\right) g^{(i)}\left(b_{k}\right)
$$

where the functions $f$ and $g$ belong to $L^{2}(\mu) \cap C^{\infty}$ and we assume that their derivatives exist at $b_{k}$. Here $\mu$ is a finite positive Borel measure supported on an infinite subset of the real line, $M_{k, i}$ are real or complex numbers such that $M_{k, N_{k}} \neq 0$, and $b_{k}, k=$ $1, \ldots, K$, are real mass points. Let $\left(\hat{B}_{n}\right)_{n=0}^{\infty}$ be the sequence of polynomials such that

$$
\operatorname{deg}\left(\hat{B}_{n}\right) \leq n, \quad\left\langle\hat{B}_{n}, p\right\rangle=0, \quad p \in \mathbb{P}_{n-1}, \quad \int\left|\hat{B}_{n}(x)\right|^{2} d \mu(x)=1 .
$$

The study of the asymptotic properties of the sequence $\left(\hat{B}_{n}\right)_{n=0}^{\infty}$ has been analyzed considering the cases 'mass points inside' or 'mass points outside' of supp $\mu$, respectively, being supp $\mu$ a compact subset of $\mathbb{R}$ or, more recently, an unbounded interval of the real line (see, for instance [7,14]). The first results in the literature about asymptotic properties for orthogonal polynomials with respect to a Sobolev type inner product like (1) appear in [15], where the authors studied the case when there is only one mass point supporting the derivatives either inside or outside $[-1,1]$ and $\mu$ is a measure in the Nevai class $M(0,1)$. Let us remind that the support of a measure of the Nevai 
class consists of $[-1,1]$ and at most a denumerable set of real mass points located outside $[-1,1]$ which, when infinitely many, may accumulate only at the end points of the above interval. In particular, if $\mu^{\prime}>0$, a.e. in $[-1,1]$, then $\mu \in M(0,1)$. In [11], using an approach based on the theory of Padé approximants, the authors obtain the outer relative asymptotics for orthogonal polynomials with respect to the Sobolev-type inner product (1) provided that $\mu$ belongs to Nevai class $M(0,1)$ and the mass points $b_{k}$ belong to $\mathbb{C} \backslash \operatorname{supp} \mu$. The same problem with the mass points in supp $\mu=[-1,1]$ was solved in [24], provided that $\mu^{\prime}(x)>0$ a.e. $x \in[-1,1]$ and $M_{k, i}$ being nonnegative constants. The asymptotics for orthogonal polynomials with respect to the Sobolev-type inner product (1) with $\mu \in M(0,1)$, some $b_{k}$ belonging supp $\mu \backslash[-1,1]$ and $M_{k, i}$ complex numbers such that $M_{k, N_{k}} \neq 0$, was solved in [2].

Other results corresponding to the study of asymptotics for orthogonal polynomials with respect to diagonal (resp. non-diagonal) Sobolev inner products with respect to measures supported on the complex plane can be found in $[1,4,6,13]$. Results related to asymptotics for extremal polynomials associated to non-diagonal Sobolev norms may be seen in $[12,18-20]$.

In this paper, we emphasize the role of the recurrence relations that the Sobolev orthogonal polynomials satisfy as well as their matrix interpretation in terms of a multiplication operator which is related to the evaluation of such a polynomial in the Jacobi matrix associated with the measure $\mu$. Based on a matrix approach, we find the connection coefficients for such sequences. Thus, an efficient algorithm is given in order to deduce those recurrence coefficients.

Notice that the main idea of $[2,24]$ and our contribution is to compare the polynomials $\left(\hat{B}_{n}\right)_{n=0}^{\infty}$ with the polynomials $\left(p_{n}\right)_{n=0}^{\infty}$ orthonormal with respect to the measure $\mu$. In order to obtain estimates of the Sobolev orthogonal polynomials $\hat{B}_{n}$ we follow, essentially, the same techniques used in these papers and we present an alternative proof for a special case of [11, Theorem 4] (see the proof of Theorem 1) based on the connection formulas.

The paper is structured as follows. Section 2 has an auxiliary character and provides some background as well as some technical lemmas in order to simplify the proof of Theorem 1 about the outer relative asymptotics of the polynomials $\left(\hat{B}_{n}\right)_{n=0}^{\infty}$. In Sect. 3 we deduce a new matrix interpretation of the recurrence relation satisfied by the polynomial sequence $\left(\hat{B}_{n}\right)_{n=0}^{\infty}$ in terms of a matrix polynomial of the Jacobi matrix associated with the sequence of orthonormal polynomials $\left(p_{n}\right)_{n=0}^{\infty}$. The analysis of the connection coefficients for such sequences constitutes a basic tool for such an approach. In Sect. 4 we give the proof of the Theorem concerning the outer relative asymptotics explained above.

\section{Background and previous results}

Let $\mu$ be a finite positive Borel measure supported on the interval $[-1,1]$ with infinitely many points at the support and let $b_{k}, k=1, \ldots, K$, be real numbers located outside $[-1,1]$ with a period. For $f$ and $g$ in $L^{2}(\mu) \cap C^{\infty}$ such that there exist the derivatives at $b_{k}$, we can introduce the Sobolev-type inner product 


$$
\langle f, g\rangle=(f, g)+\sum_{k=1}^{K} \sum_{i=0}^{N_{k}} M_{k, i} f^{(i)}\left(b_{k}\right) g^{(i)}\left(b_{k}\right),
$$

with $(f, g)=\int f(x) g(x) d \mu(x), M_{k, i} \in \mathbb{C}$, and $M_{k, N_{k}} \neq 0$. For convenience, we work with another normalization of the Sobolev orthogonal polynomials. Let $\left(\tilde{B}_{n}\right)_{n=0}^{\infty}$ be the sequence of monic polynomials of least degree such that

$$
\left\langle\tilde{B}_{n}, p\right\rangle=0, \quad p \in \mathbb{P}_{n-1},
$$

where $\mathbb{P}_{n-1}$ is the linear space of all polynomials with complex coefficients of degree less than or equal to $n-1$. The existence of $\tilde{B}_{n} \in \mathbb{P}_{n}$ for each $n \in \mathbb{Z}_{+}$follows from solving a linear system of $n$ homogeneous equations and $n+1$ unknowns. Since $\int\left|\tilde{B}_{n}(x)\right|^{2} d \mu(x)=1 / \tau_{n}^{2}>0$, we can define $\hat{B}_{n}(x)=\tau_{n} \tilde{B}_{n}(x)$ and we have a sequence $\left(\hat{B}_{n}\right)_{n=0}^{\infty}$ such that

$$
\operatorname{deg}\left(\hat{B}_{n}\right) \leq n, \quad\left\langle\hat{B}_{n}, p\right\rangle=0, \quad p \in \mathbb{P}_{n-1}, \quad \int\left|\hat{B}_{n}(x)\right|^{2} d \mu(x)=1 .
$$

It is clear that the polynomials $\hat{B}_{n}$ are not orthonormal with respect to (2), but it is possible to prove that, for $\mu$ belonging to the Nevai class $M(0,1)$ and $n$ large enough, they are equal up to constant factors $\alpha_{n}$, with $\lim _{n \rightarrow \infty} \alpha_{n}=1$. More precisely,

Lemma 1 [2, Lemma 2.2] For $\mu \in M(0,1)$, the polynomials $\hat{B}_{n}$ satisfy the conditions

(i) If $M_{k, i} \neq 0$ then $\lim _{n \rightarrow \infty} \hat{B}_{n}^{(i)}\left(b_{k}\right)=0$.

(ii) $\lim _{n \rightarrow \infty}\left\langle\hat{B}_{n}, \hat{B}_{n}\right\rangle=1$.

(iii) There exists a positive integer $n_{0}$ such that $\operatorname{deg}\left(\hat{B}_{n}\right)=n$ for all $n \geq n_{0}$.

Let $\left(p_{n}\right)_{n=0}^{\infty}$ be the sequence of orthonormal polynomials with respect to $\mu$. In what follows, we assume that either $\mu^{\prime}>0$ a.e. on the support of $\mu$ or $\mu \in M(0,1)$ with the additional assumption that none of the mass points $b_{k}$ belong to the support of the measure $\mu$ (cf. $[16,21,22]$ ), and we denote by $\kappa\left(\Pi_{n}\right)$ the leading coefficient of $\Pi_{n} \in \mathbb{P}_{n}$ with $\operatorname{deg}\left(\Pi_{n}\right)=n$. Let us consider $N=\sum_{k=1}^{K}\left(N_{k}+1\right)$ and the polynomial

$$
\omega_{N}(x):=\prod_{k=1}^{K}\left(x-b_{k}\right)^{N_{k}+1} .
$$

Let $I_{k}$ be the number of coefficients $M_{k, j}, j=0, \ldots, N_{k}$, different from 0 in (1) and let $J_{k}$ be such that $I_{k}+J_{k}=N_{k}+1$.

In order to deduce asymptotic properties for the polynomials $\hat{B}_{n}$, a successful strategy has certainly been to find orthogonality relations involving the polynomials $\hat{B}_{n} p_{n}, \omega_{N}$, and the monomials $\left(x-b_{k}\right)^{m}$, for $m \in\left\{1, \ldots, I_{k}\right\} \cup\left\{1, \ldots, J_{k}\right\}$. Concerning this issue, we have the following result. 
Lemma 2 (i) For $m=1, \ldots, I_{k}$, we have

$$
\lim _{n \rightarrow \infty} \int \frac{\hat{B}_{n}(x) p_{n}(x)}{\left(x-b_{k}\right)^{m}} d \mu(x)=0 .
$$

(ii) If $J_{k}>0$ then

$$
\lim _{n \rightarrow \infty} \int \frac{\omega_{N}(x) \hat{B}_{n}(x) p_{n-N}(x)}{\left(x-b_{k}\right)^{m}} d \mu(x)=0, \quad m=1, \ldots, J_{k}
$$

Proof It suffices to follow the proof given in [2, Lemma 3.1], making the corresponding modifications.

Lemma 3 For $n \geq n_{0}$, the polynomial $\omega_{N} \hat{B}_{n}$ has the following representation in terms of the sequence $\left(p_{n}\right)_{n=0}^{\infty}$ of orthonormal polynomials with respect to $\mu$.

$$
\omega_{N}(x) \hat{B}_{n}(x)=\sum_{j=0}^{2 N} A_{n, j} p_{n+N-j}(x), \quad A_{n, 0} \neq 0 .
$$

Moreover, $A_{n, j}$ are bounded and $A_{n, 2 N}=\frac{\kappa\left(p_{n-N}\right)}{\kappa\left(p_{n+N}\right)} \frac{1}{A_{n, 0}}\left\langle\hat{B}_{n}, \hat{B}_{n}\right\rangle \neq 0$.

Proof (5) is an immediate consequence of

$$
\int \omega_{N}(x) \hat{B}_{n}(x) p_{i}(x) d \mu(x)=\left\langle\hat{B}_{n}, \omega_{N} p_{i}\right\rangle
$$

Thus,

$$
\begin{aligned}
\sum_{i=0}^{2 N}\left|A_{n, i}\right|^{2} & =\int \omega_{N}^{2}(x)\left|\hat{B}_{n}(x)\right|^{2} d \mu(x) \leq \max _{x \in \operatorname{Supp} \mu} \omega_{N}^{2}(x), \\
A_{n, 0} & =\int \omega_{N}(x) \hat{B}_{n}(x) p_{n+N}(x) d \mu(x)=\frac{\kappa\left(\hat{B}_{n}\right)}{\kappa\left(p_{n+N}\right)}, \\
A_{n, 2 N} & =\int \omega_{N}(x) \hat{B}_{n}(x) p_{n-N}(x) d \mu(x)=\left\langle\hat{B}_{n}, \omega_{N} p_{n-N}\right\rangle \\
& =\frac{\kappa\left(p_{n-N}\right)}{\kappa\left(\hat{B}_{n}\right)}\left\langle\hat{B}_{n}, \hat{B}_{n}\right\rangle, \quad n \geq n_{0} .
\end{aligned}
$$

Therefore, the coefficients $A_{n, j}$ are bounded, $0 \leq j \leq 2 N$, and $A_{n, 0}, A_{n, 2 N}$ satisfy

$$
A_{n, 0} A_{n, 2 N}=\frac{\kappa\left(p_{n-N}\right)}{\kappa\left(p_{n+N}\right)}\left\langle\hat{B}_{n}, \hat{B}_{n}\right\rangle
$$




\section{Recurrence relations and connection coefficients}

Next, we will assume that the values $M_{k, i}$ in the inner product (2) are nonnegative real numbers. In such a way, $n_{0}=0$ in Lemma 1 and we can define the sequence of orthogonal polynomials $\left(\hat{B}_{n}\right)_{n=0}^{\infty}$ with $\operatorname{deg}\left(\hat{B}_{n}\right)=n$. Thus, it constitutes a basis of the linear space $\mathbb{P}_{n}$.

Lemma 4 The polynomial $\hat{B}_{n}$ has the following representation in terms of the sequence $\left(p_{n}\right)_{n=0}^{\infty}$ of orthonormal polynomials with respect to $\mu$.

$$
\hat{B}_{n}(x)=\sum_{j=0}^{n} \alpha_{n, j} p_{j}(x)
$$

where

$$
\begin{aligned}
\alpha_{n, n} & =\frac{\tau_{n}}{\kappa\left(p_{n}\right)}, \\
\alpha_{n, j} & =-\sum_{k=1}^{K} \sum_{i=0}^{N_{k}} M_{k, i} \hat{B}_{n}^{(i)}\left(b_{k}\right) p_{j}^{(i)}\left(b_{k}\right), \quad \text { for } 0 \leq j \leq n-1 .
\end{aligned}
$$

Proof By orthonormality of $p_{n}$, we have

$$
\alpha_{n, n}=\int \hat{B}_{n}(x) p_{n}(x) d \mu(x)=\tau_{n} \int \tilde{B}_{n}(x) p_{n}(x) d \mu(x)=\frac{\tau_{n}}{\kappa\left(p_{n}\right)} .
$$

For $0 \leq j \leq n-1$, using (3) we have

$$
\begin{aligned}
\alpha_{n, j} & =\int \hat{B}_{n}(x) p_{j}(x) d \mu(x) \\
& =\left\langle\hat{B}_{n}, p_{j}\right\rangle-\sum_{k=1}^{K} \sum_{i=0}^{N_{k}} M_{k, i} \hat{B}_{n}^{(i)}\left(b_{k}\right) p_{j}^{(i)}\left(b_{k}\right) \\
& =-\sum_{k=1}^{K} \sum_{i=0}^{N_{k}} M_{k, i} \hat{B}_{n}^{(i)}\left(b_{k}\right) p_{j}^{(i)}\left(b_{k}\right) .
\end{aligned}
$$

Lemma 5 The polynomial $p_{n}$ has the following representation in terms of the sequence $\left(\hat{B}_{n}\right)_{n=0}^{\infty}$.

$$
p_{n}(x)=\sum_{j=0}^{n} \beta_{n, j} \hat{B}_{j}(x)
$$


where

$$
\begin{aligned}
& \beta_{n, n}=\frac{\tau_{n}}{\left\langle\hat{B}_{n}, \hat{B}_{n}\right\rangle \kappa\left(p_{n}\right)}+\frac{1}{\left\langle\hat{B}_{n}, \hat{B}_{n}\right\rangle} \sum_{k=1}^{K} \sum_{i=0}^{N_{k}} M_{k, i} p_{n}^{(i)}\left(b_{k}\right) \hat{B}_{n}^{(i)}\left(b_{k}\right), \\
& \beta_{n, j}=\frac{1}{\left\langle\hat{B}_{j}, \hat{B}_{j}\right\rangle} \sum_{k=1}^{K} \sum_{i=0}^{N_{k}} M_{k, i} p_{n}^{(i)}\left(b_{k}\right) \hat{B}_{j}^{(i)}\left(b_{k}\right), \quad \text { for } 0 \leq j \leq n-1 .
\end{aligned}
$$

Proof This is a straightforward result that follows by using the same arguments as in the previous lemma.

In order to write in matrix form, we introduce the following notation.

$$
\begin{aligned}
\hat{\mathbb{B}} & =\left(\hat{B}_{0}(x), \ldots, \hat{B}_{n}(x), \ldots\right)^{T}, \\
\mathcal{P} & =\left(p_{0}(x), \ldots, p_{n}(x), \ldots\right)^{T},
\end{aligned}
$$

and $\Lambda$ being the following lower triangular infinite matrix

$$
\Lambda=\left(\begin{array}{cccc}
\alpha_{0,0} & 0 & 0 & \cdots \\
\alpha_{1,0} & \alpha_{1,1} & 0 & \ldots \\
\alpha_{2,0} & \alpha_{2,1} & \alpha_{2,2} & \ldots \\
\vdots & \vdots & \vdots & \ddots
\end{array}\right)
$$

Then $\hat{\mathbb{B}}=\Lambda \mathcal{P}$ and (6), (7) and (5), respectively, can be written in matrix form as follows.

$$
\begin{aligned}
\hat{B}_{n}(x) & =\left(\alpha_{n, 0}, \ldots, \alpha_{n, n}\right)\left(p_{0}(x), \ldots, p_{n}(x)\right)^{T} \\
p_{n}(x) & =\left(\beta_{n, 0}, \ldots, \beta_{n, n}\right)\left(\hat{B}_{0}(x), \ldots, \hat{B}_{n}(x)\right)^{T}
\end{aligned}
$$

and

$$
\begin{aligned}
\omega_{N}(x) \hat{B}_{n}(x) & =\left(A_{n, 2 N}, \ldots, A_{n, 0}\right)\left(p_{n-N}(x), \ldots, p_{n+N}(x)\right)^{T} \\
& =\left(A_{n, 2 N}, \ldots, A_{n, 0}\right) \tilde{H}\left(\hat{B}_{0}(x), \ldots, \hat{B}_{n+N}(x)\right)^{T},
\end{aligned}
$$

where $\tilde{H} \in \mathbb{R}^{(2 N+1) \times(n+N+1)}$ is the Hessenberg matrix given by

$$
\tilde{H}=\left(\begin{array}{cccccccc}
\beta_{n-N, 0} & \cdots & \beta_{n-N, n-N} & \cdots & 0 & 0 & \cdots & 0 \\
\vdots & \vdots & \vdots & \vdots & \vdots & \vdots & \vdots & \vdots \\
\beta_{n, 0} & \cdots & \beta_{n, n-N} & \cdots & \beta_{n, n} & 0 & \cdots & 0 \\
\vdots & \vdots & \vdots & \vdots & \vdots & \vdots & \ddots & \vdots \\
\beta_{n+N, 0} & \cdots & \beta_{n+N, n-N} & \cdots & \beta_{n+N, n} & \beta_{n+N, n+1} & \cdots & \beta_{n+N, n+N}
\end{array}\right) .
$$


Now, we decompose

$$
\begin{aligned}
\tilde{H}\left[\left(\begin{array}{c}
\hat{B}_{0}(x) \\
\vdots \\
\hat{B}_{n-N-1}(x) \\
0 \\
\vdots \\
0
\end{array}\right)+\left(\begin{array}{c}
0 \\
\vdots \\
0 \\
\hat{B}_{n-N}(x) \\
\vdots \\
\hat{B}_{n+N}(x)
\end{array}\right)\right] \\
=\left(\begin{array}{ccc}
\beta_{n-N, 0} & \cdots & \beta_{n-N, n-N-1} \\
\vdots & & \vdots \\
\beta_{n+N, 0} & \cdots & \beta_{n+N, n-N-1}
\end{array}\right)\left(\begin{array}{c}
\hat{B}_{0}(x) \\
\vdots \\
\hat{B}_{n-N-1}(x)
\end{array}\right)+\hat{H}\left(\begin{array}{c}
\hat{B}_{n-N}(x) \\
\vdots \\
\hat{B}_{n+N}(x)
\end{array}\right),
\end{aligned}
$$

where $\hat{H}$ is the matrix obtained from $\tilde{H}$ deleting its firts column, i.e.

$$
\hat{H}=\left(\begin{array}{ccc}
\beta_{n-N, n-N} & \cdots & 0 \\
\vdots & \ddots & \vdots \\
\beta_{n+N, n-N} & \cdots & \beta_{n+N, n+N}
\end{array}\right)
$$

Having in mind that $\omega_{N} \hat{B}_{n}(x) \in \operatorname{span}\left(\hat{B}_{n-N}, \ldots, \hat{B}_{n+N}\right)$ it follows that

$$
\left(A_{n, 2 N,} \ldots, A_{n, 0}\right)\left(\begin{array}{ccc}
\beta_{n-N, 0} & \cdots & \beta_{n-N, n-N-1} \\
\vdots & & \vdots \\
\beta_{n+N, 0} & \cdots & \beta_{n+N, n-N-1}
\end{array}\right)=(0, \ldots, 0)
$$

Finally, we obtain

$$
\omega_{N}(x) \hat{B}_{n}(x)=\left(A_{n, 2 N}, \ldots, A_{n, 0}\right) \hat{H}\left(\hat{B}_{n-N}(x), \ldots, \hat{B}_{n+N}(x)\right)^{T} .
$$

These remarks can be summarized as follows.

Proposition 1 The sequence of polynomials $\left(\hat{B}_{n}\right)_{n=0}^{\infty}$ satisfies the following recurrence relation.

$$
\omega_{N}(x) \hat{B}_{n}(x)=\sum_{j=0}^{2 N} c_{n, j} \hat{B}_{n+N-j}(x),
$$


where

$$
\left(c_{n, 2 N}, \ldots, c_{n, 0}\right)=\left(A_{n, 2 N}, \ldots, A_{n, 0}\right)\left(\begin{array}{ccc}
\beta_{n-N, n-N} & \cdots & 0 \\
\vdots & \ddots & \vdots \\
\beta_{n+N, n-N} & \cdots & \beta_{n+N, n+N}
\end{array}\right) .
$$

On the other hand, according to Lemma 3 and Lemma 4

$$
\begin{aligned}
A_{n, j} & =\int \omega_{N}(x) \hat{B}_{n}(x) p_{n+N-j}(x) d \mu(x) \\
& =\left\langle\omega_{N} \hat{B}_{n}, p_{n+N-j}\right\rangle=\left\langle\hat{B}_{n}, \omega_{N} p_{n+N-j}\right\rangle \\
& =\left\langle\sum_{l=0}^{n} \alpha_{n, l} p_{l}, \omega_{N} p_{n+N-j}\right\rangle \\
& =\sum_{l=0}^{n} \alpha_{n, l}\left\langle p_{l}, \omega_{N} p_{n+N-j}\right\rangle .
\end{aligned}
$$

But,

$$
\left\langle\omega_{N} p_{n+N-j}, p_{l}\right\rangle=\left[\omega_{N}(J)\right]_{n+N-j, l},
$$

i.e., $\left\langle\omega_{N} p_{n+N-j}, p_{l}\right\rangle$ is the entry $(n+N-j, l)$ in the $(2 N+1)$-diagonal matrix $\omega_{N}(J)$, where $J$ is the Jacobi matrix associated with the measure $\mu$, i.e. the matrix associated with the multiplication operator in terms of the orthonormal basis $\left(p_{n}\right)_{n=0}^{\infty}$.

Notice that

$$
\begin{aligned}
A_{n, 2 N} & =\sum_{l=0}^{n} \alpha_{n, l}\left[\omega_{N}(J)\right]_{n-N, l}=\sum_{l=n-2 N}^{n} \alpha_{n, l}\left[\omega_{N}(J)\right]_{n-N, l} \\
A_{n, 2 N-1} & =\sum_{l=0}^{n} \alpha_{n, l}\left[\omega_{N}(J)\right]_{n-N+1, l}=\sum_{l=n-2 N+1}^{n} \alpha_{n, l}\left[\omega_{N}(J)\right]_{n-N+1, l} \\
\vdots & \\
A_{n, 0} & =\sum_{l=0}^{n} \alpha_{n, l}\left[\omega_{N}(J)\right]_{n+N, l}=\alpha_{n, n}\left[\omega_{N}(J)\right]_{n+N, n} .
\end{aligned}
$$

Thus,

$$
\begin{aligned}
& \left(A_{n, 2 N}, \ldots, A_{n, 0}\right) \\
& =\left(\alpha_{n, n-2 N}, \ldots, \alpha_{n, n}\right)\left(\begin{array}{ccc}
{\left[\omega_{N}(J)\right]_{n-N, n-2 N}} & \cdots & 0 \\
\vdots & \ddots & \vdots \\
{\left[\omega_{N}(J)\right]_{n-N, n}} & \cdots & {\left[\omega_{N}(J)\right]_{n+N, n}}
\end{array}\right) .
\end{aligned}
$$

As a conclusion, from Proposition 1 we obtain 


$$
\begin{aligned}
& \left(c_{n, 2 N}, \ldots, c_{n, 0}\right) \\
& =\left(\alpha_{n, n-2 N}, \ldots, \alpha_{n, n}\right)\left(\begin{array}{ccc}
{\left[\omega_{N}(J)\right]_{n-N, n-2 N}} & \ldots & 0 \\
\vdots & \ddots & \vdots \\
{\left[\omega_{N}(J)\right]_{n-N, n}} & \cdots & {\left[\omega_{N}(J)\right]_{n+N, n}}
\end{array}\right) \\
& \quad \times\left(\begin{array}{ccc}
\beta_{n-N, n-N} & \cdots & \vdots \\
\vdots & \ddots & \\
\beta_{n+N, n-N} & \cdots & \beta_{n+N, n+N}
\end{array}\right) .
\end{aligned}
$$

This yields the relation between the parameters of the recurrence formula for $\left(\hat{B}_{n}\right)$ in terms of $\left(\alpha_{n, j}\right)_{j=n-2 N}^{n}$ and $\left(\beta_{n-N+k, j}\right)_{k=0}^{2 N}$.

On the other hand, from Lemma 3

$$
\omega_{N} \hat{\mathbb{B}}=H \mathcal{P} .
$$

Here $H$ denotes the $2 N+1$ banded infinite matrix with entries $h_{k, j}=A_{k, k+N-j}$ $k-N \leq j \leq k+N$, and 0 otherwise.

Given $\mathcal{C}=\left(c_{0}(x), \ldots, c_{n}(x), \ldots\right)^{T}$ and $\mathcal{D}=\left(d_{0}(x), \ldots, d_{n}(x), \ldots\right)^{T}$, we will denote by $\left(\mathcal{C}, \mathcal{D}^{T}\right)$ and $\left\langle\mathcal{C}, \mathcal{D}^{T}\right\rangle$ the infinite matrices whose entries are $\left(c_{i}(x), d_{j}(x)\right)$ and $\left\langle c_{i}(x), d_{j}(x)\right\rangle$, respectively.

Since $\left(\mathcal{P}, \mathcal{P}^{T}\right)=I$, we have

$$
\begin{aligned}
H & =\left(\omega_{N} \hat{\mathbb{B}}, \mathcal{P}^{T}\right)=\left(\hat{\mathbb{B}}, \omega_{N} \mathcal{P}^{T}\right)=\left(\hat{\mathbb{B}}, \mathcal{P}^{T} \omega_{N}(J)\right) \\
& =\left(\Lambda \mathcal{P}, \mathcal{P}^{T}\right) \omega_{N}(J)=\Lambda \omega_{N}(J) .
\end{aligned}
$$

From Proposition 1 we get

$$
\omega_{N} \hat{\mathbb{B}}=S \hat{\mathbb{B}}
$$

hence $S\left\langle\hat{\mathbb{B}}, \hat{\mathbb{B}}^{T}\right\rangle=\left\langle\omega_{N} \hat{\mathbb{B}}, \hat{\mathbb{B}}^{T}\right\rangle$. Here $S$ is the $2 N+1$ banded infinite matrix with entries $s_{k, j}=c_{k, k+N-j} k-N \leq j \leq k+N$, and 0 otherwise. But $\left\langle\omega_{N} \hat{\mathbb{B}}, \hat{\mathbb{B}}^{T}\right\rangle=\left(\omega_{N} \hat{\mathbb{B}}, \hat{\mathbb{B}}^{T}\right)=$ $\left(H \mathcal{P}, \mathcal{P}^{T} \Lambda^{T}\right)=H \Lambda^{T}$. Taking into account that $\left\langle\hat{\mathbb{B}}, \hat{\mathbb{B}}^{T}\right\rangle=D$ is a diagonal matrix according to the orthogonality of the polynomial sequence $\left(\hat{B}_{n}\right)_{n=0}^{\infty}$ with respect to our Sobolev inner product, then $S D=H \Lambda^{T}$ and we get the following result.

Proposition 2 The matrices $S$ and $\omega_{N}(J)$ satisfy the following connection relation

$$
S D=\Lambda \omega_{N}(J) \Lambda^{T} .
$$

\section{Outer relative asymptotics}

We can already provide an alternative (and more simple) proof of a well-known result about the outer relative asymptotics for the polynomials $\hat{B}_{n}$, which is a special case of [11, Theorem 4]. We denote by $T_{j}$ the $j$ th Chebyshev polynomial of the first kind 
and by $\varphi^{ \pm}(x):=x \pm \sqrt{x^{2}-1}$ with the assumption that the square root is positive for $x>1$.

Theorem 1 [11, formula (1.10)] Let $\mu$ be a finite positive Borel measure in the Nevai class $M(0,1)$, such that all the mass points $b_{k} \notin \operatorname{supp} \mu$. Then the polynomials $\left(\hat{B}_{n}\right)_{n=0}^{\infty}$ satisfy

$$
\lim _{n \rightarrow \infty} \frac{\hat{B}_{n}(x)}{p_{n}(x)}=\prod_{k=1}^{K}\left(\frac{1}{\left|\varphi^{+}\left(b_{k}\right)\right|} \frac{\left(\varphi^{+}(x)-\varphi^{+}\left(b_{k}\right)\right)^{2}}{2 \varphi^{+}(x)\left(x-b_{k}\right)}\right)^{I_{k}}
$$

uniformly on compact sets of $\overline{\mathbb{C}} \backslash$ supp $\mu$.

Proof Since $\mu \in M(0,1)$,

$$
\lim _{n \in \Lambda} \frac{\omega_{N}(x) \hat{B}_{n}(x)}{p_{n+N}(x)}=\sum_{j=0}^{2 N} A_{j}\left(\varphi^{-}(x)\right)^{j}
$$

uniformly on compact sets of $\overline{\mathbb{C}} \backslash \operatorname{supp} \mu$.

Now, we are going to show that the $A_{j}$ 's are completely determined for any sequence of nonnegative integers $\Lambda$. In order to do it, we need to obtain a factorization of the polynomial $\sum_{j=0}^{2 N} A_{j} z^{j}$.

Since $b_{k} \notin \operatorname{supp} \mu 1 /\left(x-b_{k}\right)^{i}$, are continuous functions on supp $\mu$. Hence, for a fixed $k \in\{1, \ldots, K\}$, by orthogonality, (2.4), and the weak asymptotic property (see $[2$, formula (2)]) we get

$$
\begin{aligned}
& \lim _{n \in \Lambda} \int \frac{\omega_{N}(x)}{\left(x-b_{k}\right)^{i}} \hat{B}_{n}(x) p_{n+N}(x) d \mu(x)=\frac{1}{\pi} \int_{-1}^{1} \frac{\sum_{j=0}^{2 N} A_{j} T_{j}(x)}{\left(x-b_{k}\right)^{i}} \frac{d x}{\sqrt{1-x^{2}}}=0 \\
& \quad i=1, \ldots, N_{k}+1 .
\end{aligned}
$$

According to the residue's theorem, expression (11) means that the polynomial $\sum_{j=0}^{2 N} A_{j} z^{j}$ has a zero of multiplicity at least $N_{k}+1$ at $\varphi^{-}\left(b_{k}\right)$.

On the other hand,

$$
\begin{aligned}
& \lim _{n \rightarrow \infty} \int \frac{\omega_{N}(x)}{\left(x-b_{k}\right)^{N_{k}+1}} \hat{B}_{n}(x) \frac{p_{n+N}(x)}{\left(x-b_{k}\right)^{m}} d \mu(x) \\
& =\lim _{n \rightarrow \infty} \int\left\{\sum_{j=0}^{m-1} \frac{1}{j !}\left(\frac{\omega_{N}}{\left(x-b_{k}\right)^{N_{k}+1}} \hat{B}_{n}\right)^{(j)}\left(b_{k}\right)\left(x-b_{k}\right)^{j}\right. \\
& \left.\quad+\left(x-b_{k}\right)^{m} \pi_{n+N-N_{k}-1-m}(x)\right\} \frac{p_{n+N}(x)}{\left(x-b_{k}\right)^{m}} d \mu(x) \\
& =\lim _{n \rightarrow \infty} \sum_{j=0}^{m-1} \frac{1}{j !}\left(\frac{\omega_{N}}{\left(x-b_{k}\right)^{N_{k}+1}} \hat{B}_{n}\right)^{(j)}\left(b_{k}\right) \int \frac{p_{n+N}(x)}{\left(x-b_{k}\right)^{m-j}} d \mu(x)=0,
\end{aligned}
$$


where $\pi_{n+N-N_{k}-1-m}$ is some polynomial of degree $n+N-N_{k}-1-m$ and the last equality holds as a consequence of the following two facts (cf. [2, the proof of statement (i) of Lemma 3.1. and Lemma 2.3]).

(i)

$$
\begin{aligned}
& \lim _{n \rightarrow \infty} \frac{n^{I_{k}-1-j_{1}}}{j_{1} ! p_{n+N}\left(b_{k}\right)}\left(\frac{\omega_{N}}{\left(x-b_{k}\right)^{N_{k}+1}} \hat{B}_{n}\right)^{\left(j_{1}\right)}\left(b_{k}\right) \\
& =\cdots=\lim _{n \rightarrow \infty} \frac{n^{I_{k}-1-j_{J_{k}}}}{j_{J_{k}} ! p_{n+N}\left(b_{k}\right)}\left(\frac{\omega_{N}}{\left(x-b_{k}\right)^{N_{k}+1}} \hat{B}_{n}\right)^{\left(j_{J_{k}}\right)}\left(b_{k}\right)=0,
\end{aligned}
$$

where $j_{1}<j_{2}<\cdots<j_{J_{k}}$ are the nonnegative integers corresponding to the masses $M_{k, j}=0$.

(ii)

$$
\lim _{n \rightarrow \infty} \frac{1}{n^{m-j-1}} \int \frac{p_{n+N}(x) p_{n+N}\left(b_{k}\right)}{\left(x-b_{k}\right)^{m-j}} d \mu(x)=\frac{(-1)^{m-j}}{(m-j-1) !}\left(\frac{1}{\sqrt{b_{k}^{2}-1}}\right)^{m-j},
$$

for each $b_{k}$ and $m-j>0$.

Then $\varphi^{-}\left(b_{k}\right)$ is a zero of the polynomial $\sum_{j=0}^{2 N} A_{j} z^{j}$ of multiplicity at least $N_{k}+1+I_{k}$.

From statement (ii) of Lemma 2, we have

$$
\lim _{n \rightarrow \infty} \int \frac{\omega_{N}(x) \hat{B}_{n}(x) p_{n-N}(x)}{\left(x-b_{k}\right)^{m}} d \mu(x)=0, \quad m=1, \ldots, N_{k}+1-I_{k} .
$$

As a consequence,

$$
\begin{aligned}
& \lim _{n \in \Lambda} \int \frac{\omega_{N}(x) \hat{B}_{n}(x) p_{n-N}(x)}{\left(x-b_{k}\right)^{m}} d \mu(x) \\
& \quad=\frac{1}{\pi} \int_{-1}^{1} \frac{\sum_{j=0}^{2 N} A_{j} T_{2 N-j}(x)}{\left(x-b_{k}\right)^{m}} \frac{d x}{\sqrt{1-x^{2}}}=0, \quad m=1, \ldots, N_{k}+1-I_{k} .
\end{aligned}
$$

Hence, (14) means that $\varphi^{+}\left(b_{k}\right)$ is a zero of the polynomial $\sum_{j=0}^{2 N} A_{j} z^{j}$ of multiplicity at least $N_{k}+1-I_{k}$.

Therefore, $\sum_{j=0}^{2 N} A_{j} z^{j}$ has the following factorization

$$
\sum_{j=0}^{2 N} A_{j} z^{j}=A_{2 N} \prod_{k=1}^{K}\left(z-\varphi^{-}\left(b_{k}\right)\right)^{N_{k}+1+I_{k}}\left(z-\varphi^{+}\left(b_{k}\right)\right)^{N_{k}+1-I_{k}},
$$


and for $z=\varphi^{-}(x)$ we obtain

$$
\sum_{j=0}^{2 N} A_{j}\left(\varphi^{-}(x)\right)^{j}=A_{2 N} \prod_{k=1}^{K}\left(\varphi^{-}(x)-\varphi^{-}\left(b_{k}\right)\right)^{N_{k}+1+I_{k}}\left(\varphi^{-}(x)-\varphi^{+}\left(b_{k}\right)\right)^{N_{k}+1-I_{k}}
$$

If $x$ tends to infinity, then we find $A_{0}=A_{2 N} \prod_{k=1}^{K}\left(\varphi^{-}\left(b_{k}\right)\right)^{2 I_{k}}$, and having in mind that $A_{0} A_{2 N}=\frac{1}{2^{2 N}}$, we can deduce that

$$
A_{2 N}=\frac{\prod_{k=1}^{K}\left|\varphi^{+}\left(b_{k}\right)\right|^{I_{k}}}{2^{N}}
$$

and this last equation determines completely $A_{j}$ for any sequence of nonnegative integers $\Lambda$. Also, from (10) and (15) we can deduce that

$$
\begin{aligned}
& \lim _{n \rightarrow \infty} \frac{\omega_{N}(x) \hat{B}_{n}(x)}{p_{n+N}(x)} \\
& =\frac{\prod_{k=1}^{K}\left|\varphi^{+}\left(b_{k}\right)\right|^{I_{k}}}{2^{N}} \prod_{k=1}^{K}\left(\varphi^{-}(x)-\varphi^{-}\left(b_{k}\right)\right)^{N_{k}+1+I_{k}}\left(\varphi^{-}(x)-\varphi^{+}\left(b_{k}\right)\right)^{N_{k}+1-I_{k}} \\
& =\frac{1}{2^{N}} \prod_{k=1}^{K}\left|\varphi^{+}\left(b_{k}\right)\right|^{I_{k}}\left(\varphi^{-}(x)-\varphi^{-}\left(b_{k}\right)\right)^{N_{k}+1+I_{k}}\left(\varphi^{-}(x)-\varphi^{+}\left(b_{k}\right)\right)^{N_{k}+1-I_{k}} \\
& =\frac{1}{2^{N}} \prod_{k=1}^{K} \frac{\left|\varphi^{+}\left(b_{k}\right)\right|^{I_{k}}\left(\varphi^{-}(x)-\varphi^{-}\left(b_{k}\right)\right)^{I_{k}}}{\left(\varphi^{-}(x)-\varphi^{+}\left(b_{k}\right)\right)^{I_{k}}}\left(\left(\varphi^{-}(x)-\varphi^{-}\left(b_{k}\right)\right)\left(\varphi^{-}(x)-\varphi^{+}\left(b_{k}\right)\right)\right)^{N_{k}+1} .
\end{aligned}
$$

Finally, taking into account the outer ratio asymptotics for orthonormal polynomials associated with measures in the Nevai class, as well as the fact that

$$
\omega_{N}(x)=\frac{1}{2^{N}}\left(\varphi^{+}(x)\right)^{N} \prod_{k=1}^{K}\left(\left(\varphi^{-}(x)\right)^{2}-2 b_{k} \varphi^{-}(x)+1\right)^{N_{k}+1},
$$

on compact subsets of $\overline{\mathbb{C}} \backslash$ supp $\mu$ we get

$$
\lim _{n \rightarrow \infty} \frac{\hat{B}_{n}(x)}{p_{n}(x)}=\prod_{k=1}^{K}\left(\frac{1}{\left|\varphi^{+}\left(b_{k}\right)\right|} \frac{\left(\varphi^{+}(x)-\varphi^{+}\left(b_{k}\right)\right)^{2}}{2 \varphi^{+}(x)\left(x-b_{k}\right)}\right)^{I_{k}}
$$

Remark 1 When $b_{k} \in \operatorname{supp} \mu, k=1, \ldots, K$, for instance, $\left\{a_{k}\right\}_{k=1}^{K_{1}}$ and $\left\{b_{j}^{\prime}\right\}_{j=1}^{K_{2}}$ are the mass points of $\mu$ on $[-1,1]$ and the mass points of $\mu$ on supp $\mu \backslash[-1,1]$, respectively, for $k=1, \ldots, K_{1}$ and $j=1, \ldots, K_{2}, K=K_{1}+K_{2}$, it was proved in [2, Theorem 
3.1, (i)] that for $\mu \in M(0,1)$ the following outer relative asymptotics holds uniformly on compact subsets of $\overline{\mathbb{C}} \backslash \operatorname{supp} \mu$.

$$
\lim _{n \rightarrow \infty} \frac{\hat{B}_{n}(x)}{q_{n}(x)}=\prod_{k=1}^{K_{2}}\left(\frac{1}{\left|\varphi^{+}\left(b_{k}^{\prime}\right)\right|} \frac{\left(\varphi^{+}(x)-\varphi^{+}\left(b_{k}^{\prime}\right)\right)^{2}}{2 \varphi^{+}(x)\left(x-b_{k}^{\prime}\right)}\right)^{I_{k}}
$$

where $\left(q_{n}\right)_{n=0}^{\infty}$ is the sequence of orthonormal polynomials with respect to the measure $v \in M(0,1)$ defined by $v=\mu-\sum_{k=1}^{K_{1}} \mu\left(\left\{a_{k}\right\}\right) \delta_{a_{k}}-\sum_{k=1}^{K_{2}} \mu\left(\left\{b_{k}^{\prime}\right\}\right) \delta_{b_{k}^{\prime}}$.

Even though in the previous proof we follow the ideas of the proof given in [2, Theorem 3.1], it is worthwhile to point out that the existing difference between the arguments of both proofs is the use of Lemma 2, which is necessary for us since we do not consider the mass points of $\mu$ inside supp $\mu$.

Remark 2 It would be interesting to analyze two open problems concerning the inner strong asymptotics (resp. the outer relative asymptotics) for orthogonal polynomials with respect to Sobolev-type inner products when $K=\infty$ and $\mu$ belongs to the Szegó (resp. Nevai) class. Notice that when you consider standard inner products associated with measures $\mu$ belonging to the Szegó class $\mathcal{S}$, i.e. $\frac{\ln \mu^{\prime}}{\left(1-x^{2}\right)^{1 / 2}} \in L^{1}([-1,1])$, and you add a denumerable set of mass points outside the interval $[-1,1]$ which accumulate at the ends of the interval, assuming that these mass points satisfy Blaschke's condition, then inner strong asymptotics for the corresponding sequence of standard orthonormal polynomials has been obtained in [17]. On the other hand, if $\mu^{\prime}>0$ a.e. in $[-1,1]$ and you add an infinite number of mass points outside the interval $[-1,1]$, then the new measure belongs to the Nevai class, but nothing is said about the outer relative asymptotics for the new sequence of standard orthonormal polynomials (see [5]). Thus, this last situation needs a deep analysis. Of course, in the Sobolev case, when you add a denumerable set of mass points at the non standard component of the inner product, the asymptotic behavior (inner strong asymptotics for measures in the Szegó class and outer relative asymptotics in the Nevai class, respectively) of the corresponding orthonormal polynomials constitute more intriguing problems than those pointed out above.

Acknowledgments The authors thank the referees for their careful revision of the manuscript. Their helpful comments and suggestions contributed to improve substantially the presentation of the manuscript.

Open Access This article is distributed under the terms of the Creative Commons Attribution License which permits any use, distribution, and reproduction in any medium, provided the original author(s) and the source are credited.

\section{References}

1. Alfaro, M., Marcellán, F., Rezola, M.L., Ronveaux, A.: Sobolev-type orthogonal polynomials: the non-diagonal case. J. Approx. Theory 83, 266-287 (1995)

2. Alvarez Rocha, I., Salto, L.: Asymptotics of polynomials orthogonal with respect to a discrete-complex Sobolev inner product. J. Comput. Appl. Math. 178, 1-19 (2005) 
3. Baratchart, I., Martínez-Finkelshtein, A., Jiménez, D., Lubinsky, D.S., Mhaskar, H.N., Pritsker, I., Putinar, M., Stylianopoulus, M., Totik, V., Varju, P., Xu, Y.: Open problems in constructive function theory. Electron. Trans. Numer. Anal. 25, 511-525 (2006)

4. Branquinho, A., Foulquié, A., Marcellán, F.: Asymptotic behavior of Sobolev type orthogonal polynomials on a rectifiable Jordan curve or arc. Constr. Approx. 18, 161-182 (2002)

5. Denisov, S.A.: On Rakhmanov's theorem for Jacobi matrices. Proc. Am. Math. Soc. 132, 847-852 (2004)

6. Dueñas, H., Marcellán, F.: Asymptotic behaviour of Laguerre-Sobolev type orthogonal polynomials: a nondiagonal case. J. Comput. Appl. Math. 235, 998-1007 (2010)

7. Dueñas, H., Huertas, E.J., Marcellán, F.: Asymptotic properties of Laguerre-Sobolev type orthogonal polynomials. Numer. Algorithms 60, 51-73 (2011)

8. Foulquié, A., Marcellán, F., Pan, K.: Asymptotic behavior of Sobolev-type orthogonal polynomials on the unit circle. J. Approx. Theory 100, 345-363 (1999)

9. López Lagomasino, G.: Relative asymptotics of orthogonal polynomials on the real axis, Mat. Sb. 137(179), 266-287 (1988) (English translation: Math. USSR-Sb. 65, 505-527 (1990))

10. López Lagomasino, G.: Asymptotics of polynomials orthogonal with respect to varying measures. Constr. Approx. 5, 199-219 (1989)

11. López Lagomasino, G., Marcellán, F., Van Assche, W.: Relative symptotics for polynomials orthogonal with respect to a discrete Sobolev inner product. Constr. Approx. 11, 107-137 (1995)

12. López Lagomasin, G., Pérez Izquierdo, I., Pijeira, H.: Asymptotic of extremal polynomials in the complex plane. J. Approx. Theory 137, 226-237 (2005)

13. López Lagomasino, G., Pijeira, H.: Zero location and n-th root asymptotics of Sobolev orthogonal polynomials. J. Approx. Theory 99, 30-43 (1999)

14. Marcellán, F., Moreno-Balcázar, J.J.: Asymptotics and zeros of Sobolev orthogonal polynomials on unbounded supports. Acta Appl. Math. 94, 163-192 (2006)

15. Marcellán, F., Van Assche, W.: Relative asymptotics for orthogonal polynomials with a Sobolev inner product. J. Approx. Theory 72, 193-209 (1992)

16. Nevai, P.: Orthogonal Polynomials. Members of American Mathematical Socirty, vol. 213. American Mathematical Society, Providence (1979)

17. Peherstorfer, F., Yuditskii, P.: Asymptotics of orthonormal polynomials in the presence of a denumerable set of mass points. Proc. Am. Math. Soc. 129, 3213-3220 (2001)

18. Portilla, A., Rodríguez, J.M., Tourís, E.: The multiplication operator, zero location and asymptotic for non-diagonal Sobolev norms. Acta Appl. Math. 111, 205-218 (2010)

19. Portilla, A., Quintana, Y., Rodríguez, J.M., Tourís, E.: Zero location and asymptotic behavior for extremal polynomials with non-diagonal Sobolev norms. J. Approx. Theory 162, 2225-2242 (2010)

20. Portilla, A., Quintana, Y., Rodríguez, J.M., Tourís, E.: Concerning asymptotic behavior for extremal polynomials associated to non-diagonal Sobolev norms. J. Funct. Spaces Appl. 2013, 1-11 (2013, article ID 628031)

21. Rakhmanov, E.A.: On the asymptotics of the ratio of orthogonal polynomials. Mat. Sb. 103(145), 271-291 (1977) (English translation: Math. USSR-Sb. 32, 199-213 (1977))

22. Rakhmanov, E.A.: On the asymptotics of the ratio of orthogonal polynomials II. Mat. Sb. 118(160), 271-291 (1982) (English translation: Math. USSR-Sb. 46, 105-117 (1983))

23. Rakhmanov, E.A.: On the asymptotics of polynomials orthogonal on the circle with weights not satisfying Szegő's condition. Mat. Sb. 118(172), 151-169 (1986) (English translation: Math. USSRSb. 58, 149-167 (1987))

24. Rocha, I.A., Marcellán, F., Salto, L.: Relative asymptotics and Fourier Series of orthogonal polynomials with a discrete Sobolev inner product. J. Approx. Theory 121, 336-356 (2003)

25. Stahl, H., Totik, V.: General Orthogonal Polynomials. Cambridge University Press, Cambridge (1992)

26. Szegő, G.: Orthogonal Polynomials. Colloquium Publications, American Mathematical Society, vol. 23, 4th edn. American Mathematical Society, Providence (1975) 